Sādhanā Vol. 39, Part 3, June 2014, pp. 607-623. (C) Indian Academy of Sciences

\title{
An exact dynamic stiffness matrix for axially loaded double-beam systems
}

\author{
LI XIAOBIN，XU SHUANGXI，WU WEIGUO and LI JUN*
}

School of Transportation, Wuhan University of Technology, Wuhan, People's Republic of China

e-mail: LJY60023@yahoo.com

MS received 3 February 2012; revised 17 July 2013; accepted 10 October 2013

\begin{abstract}
An exact dynamic stiffness method is presented in this paper to determine the natural frequencies and mode shapes of the axially loaded double-beam systems, which consist of two homogeneous and prismatic beams with a distributed spring in parallel between them. The effects of the axial force, shear deformation and rotary inertia are considered, as shown in the theoretical formulation. The dynamic stiffness influence coefficients are formulated from the governing differential equations of the axially loaded double-beam system in free vibration by using the Laplace transform method. An example is given to demonstrate the effectiveness of this method, in which ten boundary conditions are investigated and the effect of the axial force on the natural frequencies and mode shapes of the double-beam system are further discussed.
\end{abstract}

Keywords. Double-beam system; Timoshenko beam; axial force; dynamic stiffness matrix; free vibration.

\section{Introduction}

Beams are widely used in various structures and mechanical equipments. Free and forced vibrations of the uniform single Bernoulli-Euler and Timoshenko beams with different boundary conditions have been investigated extensively by many authors. However, there are only a few works to study the dynamic behaviour of double-beam system which consists of two parallel beams of uniform properties with a uniformly distributed spring between them. Currently, this double-beam system is of technological interest perhaps due to its potential use in various areas of technology, like aerospace and automobile industry. The double-beam system can be regarded as an ideal model of a complex continuous system consisting of two one-dimensional structures connected by an elastic layer of a Winkler type, or as an approximate model of the sandwich beams. In addition, it can also be used as a continuous dynamic vibration absorber if one beam is regarded as a principal system and another beam as well as the coupling springs as an auxiliary system.

*For correspondence 
Seelig \& Hoppmann (1964) first presented the development and solution of the differential equations of motion of a system of elastically connected parallel beams. Vibration experiments were performed to determine the degree of applicability of the theory. Kessel (1966) investigated the resonances of a double-beam system composed of two parallel, simply supported beams that were elastically connected and subjected to a moving load. Rao (1974) derived the differential equations governing the flexural vibrations of elastically connected parallel beams with the effects of shear deformation and rotary inertia considered. Chonan (1976) investigated the dynamical behaviours of two beams connected with a set of independent springs and subjected to an impulsive load. Hamada et al (1983) analysed the free and forced vibrations of a system of two elastically connected parallel beams having unequal masses and unequal flexural rigidities by using a generalized method of the finite integral transformation and the Laplace transformation. Aida et al (1992) presented a beam-type dynamic vibration absorber which was composed of an absorbing beam under the same boundary conditions as the main beam and a uniformly distributed, connecting spring and damper between the main beam and absorbing beam. Chen \& Sheu (1994) studied analytically the dynamic characteristics of two parallel damped Timoshenko beams, connected to each other by the springs and dashpots uniformly distributed along the beam length. Chen \& Lin (1998) presented the structural analysis and optimal design of a dynamic absorbing beam which was attached to the main beam with a viscoelastic layer or other mechanism of the similar effect. Vu et al (2000) presented an exact method for solving the vibration of a double-beam system subject to a harmonic excitation. The Bernoulli-Euler theory was used for analysing the transverse vibrations of the beams. Oniszczuk (2000) discussed the free transverse vibrations of two simply supported Bernoulli-Euler beams connected by a Winkler elastic layer. Oniszczuk (2003) tried to analyse the undamped forced transverse vibrations of an elastically connected double-beam system in the case of simply supported end condition. Abu-Hilal (2006) studied the dynamic response of a double Bernoulli-Euler beam system traversed by a constant moving load, where the two beams were homogeneous, isotropic, simply supported and connected continuously by a viscoelastic layer. On the basis of the Bernoulli-Euler beam theory, Zhang et al (2008) investigated the free transverse vibration and buckling of a double-beam system under a compressive axial loading, where the two beams were simply supported and continuously joined by a Winkler elastic layer. Balkaya et al (2010) carried out the free vibration analyses of a parallel placed twin pipe system simulated by the hinged-hinged and fixed-fixed Bernoulli-Euler beams resting on a Winkler elastic soil. Palmeri \& Adhikari (2011) presented a Galerkin-type state-space approach for studying the transverse vibrations of double-beam systems which was made of two outer elastic Bernoulli-Euler beams continuously joined by an inner viscoelastic layer.

Some authors have investigated the dynamic characteristics of the elastically connected double-beam system, and only a few of them have considered the two non-identical beams and the effects of shear deformation and rotary inertia. Further, even fewer papers about the free vibration of the double-beam systems considering the effects of the axial force, shear deformation and rotary inertia in a unitary manner can be searched currently. Usually the natural frequencies of the axially loaded beams are required in the design of aerospace structures. Especially, the earlier studies have shown that the axial force has a greater effect on the natural frequencies and mode shapes than the other factors, such as the shear deformation and/or rotary inertia. Therefore, the general vibration analysis of an elastically connected double-beam system is carried out and some results are presented in this paper.

First, the coupled governing equations of motion of the axially loaded double-beam system with shear deformation and rotary inertia included are derived by using Hamilton's principle. Then the dynamic stiffness influence coefficients of the axially loaded double-beam system are 
formulated from its governing differential equations of motion by employing the Laplace transform method. Since the dynamic stiffness matrix is based on the closed-form analytical solutions of the governing differential equations, this exact nature makes the present method very useful when higher frequencies and better accuracies of the results are required.Also the exact results can be used as benchmark of traditional finite element and other approximate methods. Finally, the influence of the axial force on the natural frequencies and mode shapes of a double-beam system is intensively studied by a particular example for demonstrations and discussions.

\section{Mathematical formulation}

The double-beam system shown in figure 1 consists of an upper beam and a lower beam, with distributed springs in parallel between them. These two beams are assumed to be homogeneous, prismatic and of the same length $L$.

With the effects of shear distortion and rotary inertia of the beams considered, the kinetic and strain energies of this elastically connected double-beam system can be given by

$$
\begin{gathered}
T=\frac{1}{2} \int_{0}^{L} \rho_{1} A_{1}\left(\dot{w}_{1}\right)^{2} d x+\frac{1}{2} \int_{0}^{L} \rho_{1} I_{1}\left(\dot{\theta}_{1}\right)^{2} d x+\frac{1}{2} \int_{0}^{L} \rho_{2} A_{2}\left(\dot{w}_{2}\right)^{2} d x+\frac{1}{2} \int_{0}^{L} \rho_{2} I_{2}\left(\dot{\theta}_{2}\right)^{2} d x \\
U=\frac{1}{2} \int_{0}^{L} k_{1} G_{1} A_{1}\left(w_{1}^{\prime}-\theta_{1}\right)^{2} d x+\frac{1}{2} \int_{0}^{L} E_{1} I_{1}\left(\theta_{1}^{\prime}\right)^{2} d x+\frac{1}{2} \int_{0}^{L} k_{2} G_{2} A_{2}\left(w_{2}^{\prime}-\theta_{2}\right)^{2} d x \\
+\frac{1}{2} \int_{0}^{L} E_{2} I_{2}\left(\theta_{2}^{\prime}\right)^{2} d x+\frac{1}{2} \int_{0}^{L} k\left(w_{1}-w_{2}\right)^{2} d x
\end{gathered}
$$

where $w_{i}(x, t)$ and $\theta_{i}(x, t)$ represent the deflection and normal rotation of a beam, respectively; $\rho_{i}$ represents the mass density per unit volume of a beam; $A_{i}$ and $I_{i}$ represent the area and second moment of area of a beam section; $E_{i}$ and $G_{i}$ represent the Young's and shear moduli of a beam material; $k_{i}$ represents the shear correction factor of a beam; $k$ represents the spring coefficient per unit beam length. The subscripts 1 and 2 in Equation (1) are referred to the upper and lower beams, respectively. $(\cdot)=d / d t,\left(^{\prime}\right)=d / d x$.

The work done by the axial forces is:

$$
W=\frac{1}{2} \int_{0}^{L} P_{1}\left(w_{1}^{\prime}\right)^{2} d x+\frac{1}{2} \int_{0}^{L} P_{2}\left(w_{2}^{\prime}\right)^{2} d x,
$$

\section{upper beam}

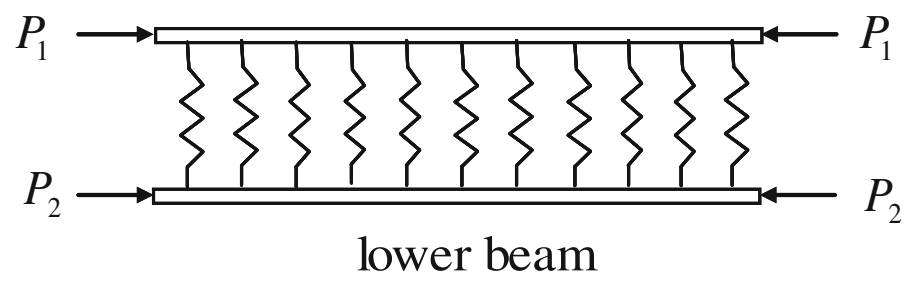

Figure 1. An axially-loaded double-beam system. 
where $P_{i}$ is a constant compressive axial force acting through the centroid of the cross-section of a beam. $P_{i}$ can be positive or negative.

The boundary value problem, consisting of the governing differential equations and the boundary conditions, can be derived conveniently by means of the extended Hamilton's principle, which can be stated in the form

$$
\begin{aligned}
& \int_{t_{1}}^{t_{2}}(\delta T-\delta U+\delta W) d t=0 \\
& \delta_{w_{1}}=\delta_{w_{2}}=\delta \theta_{1}=\delta \theta_{2}=0 \text { at } t=t_{1}, t_{2} .
\end{aligned}
$$

Substitute Equations (1a)-(1c) into Equation (2), and perform the variational operations. The following governing equations can be obtained

$$
\begin{gathered}
-\rho_{1} A_{1} \ddot{w}_{1}+k_{1} G_{1} A_{1} w_{1}^{\prime \prime}-k_{1} G_{1} A_{1} \theta_{1}^{\prime}-k\left(w_{1}-w_{2}\right)-P_{1} w_{1}^{\prime \prime}=0 \\
-\rho_{2} A_{2} \ddot{w}_{2}+k_{2} G_{2} A_{2} w_{2}^{\prime \prime}-k_{2} G_{2} A_{2} \theta_{2}^{\prime}+k\left(w_{1}-w_{2}\right)-P_{2} w_{2}^{\prime \prime}=0 \\
-\rho_{1} I_{1} \ddot{\theta}_{1}+k_{1} G_{1} A_{1} w_{1}^{\prime}-k_{1} G_{1} A_{1} \theta_{1}+E_{1} I_{1} \theta_{1}^{\prime \prime}=0 \\
-\rho_{2} I_{2} \ddot{\theta}_{2}+k_{2} G_{2} A_{2} w_{2}^{\prime}-k_{2} G_{2} A_{2} \theta_{2}+E_{2} I_{2} \theta_{2}^{\prime \prime}=0 .
\end{gathered}
$$

The boundary conditions at the ends of this double-beam are

$$
\begin{gathered}
{\left[-k_{1} G_{1} A_{1} w_{1}^{\prime}+k_{1} G_{1} A_{1} \theta_{1}+P_{1} w_{1}^{\prime}\right] \delta w_{1}=0} \\
{\left[-k_{2} G_{2} A_{2} w_{2}^{\prime}+k_{2} G_{2} A_{2} \theta_{2}+P_{2} w_{2}^{\prime}\right] \delta w_{2}=0} \\
{\left[-E_{1} I_{1} \theta_{1}^{\prime}\right] \delta \theta_{1}=0} \\
{\left[-E_{2} I_{2} \theta_{2}^{\prime}\right] \delta \theta_{2}=0 .}
\end{gathered}
$$

\section{Exact dynamic stiffness matrix}

The Laplace transform $\bar{f}(s)$ of a function of time $f(t)$ is defined by

$$
L[f(s)]=\bar{f}(s)=\int_{0}^{\infty} e^{-s t} f(t) d t
$$

where $s$ stands for the Laplace transform parameter.

Assume zero initial conditions, the partial differential Equations (3a)-(3d) can be reduced to the following equations

$$
\begin{gathered}
-\rho_{1} A_{1} s^{2} \bar{w}_{1}+k_{1} G_{1} A_{1} \bar{w}_{1}^{\prime \prime}-k_{1} G_{1} A_{1} \bar{\theta}_{1}^{\prime}-k\left(\bar{w}_{1}-\bar{w}_{2}\right)-P_{1} \bar{w}_{1}^{\prime \prime}=0, \\
-\rho_{2} A_{2} s^{2} \bar{w}_{2}+k_{2} G_{2} A_{2} \bar{w}_{2}^{\prime \prime}-k_{2} G_{2} A_{2} \bar{\theta}_{2}^{\prime}+k\left(\bar{w}_{1}-\bar{w}_{2}\right)-P_{2} \bar{w}_{2}^{\prime \prime}=0, \\
-\rho_{1} I_{1} s^{2} \bar{\theta}_{1}+k_{1} G_{1} A_{1} \bar{w}_{1}^{\prime}-k_{1} G_{1} A_{1} \bar{\theta}_{1}+E_{1} I_{1} \bar{\theta}_{1}^{\prime \prime}=0,
\end{gathered}
$$




$$
-\rho_{2} I_{2} s^{2} \bar{\theta}_{2}+k_{2} G_{2} A_{2} \bar{w}_{2}^{\prime}-k_{2} G_{2} A_{2} \bar{\theta}_{2}+E_{2} I_{2} \bar{\theta}_{2}^{\prime \prime}=0,
$$

where $\bar{w}_{1}(x, s), \bar{w}_{2}(x, s), \bar{\theta}_{1}(x, s)$ and $\bar{\theta}_{2}(x, s)$ are the Laplace transform of $w_{1}(x, t), w_{2}(x, t)$, $\theta_{1}(x, t)$ and $\theta_{2}(x, t)$, respectively.

Equations (6a)-(6d) can be combined to arrive at the following decoupled equation of motion

$$
\left\{\eta_{4} D^{8}+\eta_{3} D^{6}+\eta_{2} D^{4}+\eta_{1} D^{2}+\eta_{0}\right\} \bar{u}=0
$$

where

$$
\begin{aligned}
& D=d / d x \\
& \bar{u}=\bar{w}_{1}, \quad \bar{w}_{2}, \quad \bar{\theta}_{1} \quad \text { or } \quad \bar{\theta}_{2} \\
& \eta_{4}=E_{1} I_{1} E_{2} I_{2}\left(k_{1} G_{1} A_{1}-P_{1}\right)\left(k_{2} G_{2} A_{2}-P_{2}\right) \\
& \eta_{3}=-E_{1} I_{1} E_{2} I_{2} k_{1} G_{1} A_{1} k-E_{1} I_{1} E_{2} I_{2} k_{2} G_{2} A_{2} k+E_{1} I_{1} E_{2} I_{2} k P_{1}+E_{2} I_{2} k_{1} G_{1} A_{1} k_{2} G_{2} A_{2} P_{1} \\
& +E_{1} I_{1} E_{2} I_{2} k P_{2}+E_{1} I_{1} k_{1} G_{1} A_{1} k_{2} G_{2} A_{2} P_{2}-E_{2} I_{2} k_{1} G_{1} A_{1} P_{1} P_{2}-E_{1} I_{1} k_{2} G_{2} A_{2} P_{1} P_{2} \\
& -I_{1} I_{2}\left(E_{2}\left(E_{1} A_{1}+k_{1} G_{1} A_{1}-P_{1}\right)\left(k_{2} G_{2} A_{2}-P_{2}\right) \rho_{1}\right. \\
& \left.+E_{1}\left(k_{1} G_{1} A_{1}-P_{1}\right)\left(E_{2} A_{2}+k_{2} G_{2} A_{2}-P_{2}\right) \rho_{2}\right) s^{2} \\
& \eta_{2}=-E_{2} I_{2} k_{1} G_{1} A_{1}^{2}\left(-k_{2} G_{2} A_{2}+P_{2}\right) \rho_{1} s^{2}+A_{1}\left(k _ { 1 } G _ { 1 } \left(-E_{2} I_{2} k\left(P_{1}+P_{2}\right)\right.\right. \\
& \left.+k_{2} G_{2} A_{2}\left(E_{1} I_{1} k+E_{2} I_{2} k+P_{1} P_{2}\right)\right)+\left(I_{1}\left(E_{1}+k_{1} G_{1}\right)\left(E_{2} I_{2} k-k_{2} G_{2} A_{2} P_{2}\right) \rho_{1}\right. \\
& \left.+k_{1} G_{1}\left(E_{1} I_{1}\left(I_{2} k+k_{2} G_{2} A_{2}^{2}\right)+I_{2} P_{1}\left(-A_{2}\left(E_{2}+k_{2} G_{2}\right)+P_{2}\right)\right) \rho_{2}\right) s^{2} \\
& +I_{1} I_{2}\left(E_{2}\left(k_{2} G_{2} A_{2}-P_{2}\right) \rho_{1}^{2}+\left(E_{1}+k_{1} G_{1}\right)\left(A_{2}\left(E_{2}+k_{2} G_{2}\right)-P_{2}\right) \rho_{1} \rho_{2}\right. \\
& \left.\left.+A_{2} E_{1} k_{1} G_{1} \rho_{2}^{2}\right) s^{4}\right)+I_{1}\left(-E_{1} k_{2} G_{2} A_{2} k\left(P_{1}+P_{2}\right)-\left(-k_{2} G_{2} A_{2}\left(E_{2} I_{2} k+P_{1} P_{2}\right) \rho_{1}\right.\right. \\
& \left.-A_{2} E_{1} I_{2} k\left(E_{2}+k_{2} G_{2}\right) \rho_{2}+A_{2}^{2} E_{1} k_{2} G_{2} P_{1} \rho_{2}+I_{2} k\left(P_{1}+P_{2}\right)\left(E_{2} \rho_{1}+E_{1} \rho_{2}\right)\right) s^{2} \\
& \left.-I_{2} P_{1} \rho_{2}\left(-P_{2} \rho_{1}+A_{2}\left(E_{2} \rho_{1}+k_{2} G_{2} \rho_{1}+E_{1} \rho_{2}\right)\right) s^{4}\right) \\
& \eta_{1}=k_{1} G_{1} A_{1} k_{2} G_{2} A_{2} k\left(P_{1}+P_{2}\right)-\left(k_{2} G_{2} A_{2} k\left(E_{1}+k_{1} G_{1}\right) A_{1} \rho_{1} I_{1}\right. \\
& +k_{1} G_{1} A_{1}^{2}\left(E_{2} I_{2} k-k_{2} G_{2} A_{2} P_{2}\right) \rho_{1}-k_{1} G_{1} A_{1}\left(-I_{2} k\left(E_{2}+k_{2} G_{2}\right) A_{2}+k_{2} G_{2} A_{2}^{2} P_{1}\right. \\
& \left.\left.+k\left(P_{1}+P_{2}\right)\right) \rho_{2} I_{2}+k_{2} G_{2} A_{2} k\left(-\left(P_{1}+P_{2}\right) \rho_{1} I_{1}+E_{1} \rho_{2} A_{2}\right)\right) s^{2} \\
& -\left(A_{1} I_{1}\left(E_{2} I_{2} k-k_{2} G_{2} A_{2} P_{2}\right) \rho_{1}^{2}+\left(A_{1} I_{1}\left(E_{1}+k_{1} G_{1}\right)\left(I_{2} k+k_{2} G_{2} A_{2}^{2}\right)\right.\right. \\
& +k_{1} G_{1} A_{1}^{2} I_{2}\left(A_{2}\left(E_{2}+k_{2} G_{2}\right)-P_{2}\right)-I_{1}\left(-A_{2} I_{2} k\left(E_{2}+k_{2} G_{2}\right)+k_{2} G_{2} A_{2}^{2} P_{1}\right. \\
& \left.\left.\left.+I_{2} k\left(P_{1}+P_{2}\right)\right)\right) \rho_{1} \rho_{2}+A_{2} I_{2}\left(E_{1} I_{1} k-k_{1} G_{1} A_{1} P_{1}\right) \rho_{2}^{2}\right) s^{4} \\
& +\rho_{1} I_{1} \rho_{2} I_{2}\left(A_{1}\left(A_{2}\left(E_{2}+G_{2} k_{2}\right)-P_{2}\right) \rho_{1}+A_{1}\left(E_{1}+G_{1} k_{1}\right) \rho_{2} A_{2}-P_{1} \rho_{2} A_{2}\right) s^{6} \\
& \eta_{0}=-s^{2}\left(-k_{1} G_{1} A_{1}-\rho_{1} I_{1} s^{2}\right)\left(-k_{2} G_{2} A_{2}-\rho_{2} I_{2} s^{2}\right)\left(-k\left(\rho_{1} A_{1}+\rho_{2} A_{2}\right)-\rho_{1} A_{1} \rho_{2} A_{2} s^{2}\right) .
\end{aligned}
$$

The general solutions to Equation (7) are given by

$$
\begin{aligned}
\bar{w}_{1}(x, s)= & \bar{A}_{1} e^{\kappa_{1} x}+\bar{A}_{2} e^{-\kappa_{1} x}+\bar{A}_{3} e^{\kappa_{2} x}+\bar{A}_{4} e^{-\kappa_{2} x}+\bar{A}_{5} e^{\kappa_{3} x}+\bar{A}_{6} e^{-\kappa_{3} x} \\
& +\bar{A}_{7} e^{\kappa_{4} x}+\bar{A}_{8} e^{-\kappa_{4} x}=\sum_{j=1}^{4}\left(\bar{A}_{2 j-1} e^{\kappa_{j} x}+\bar{A}_{2 j} e^{-\kappa_{j} x}\right) \\
\bar{w}_{2}(x, s)= & \bar{B}_{1} e^{\kappa_{1} x}+\bar{B}_{2} e^{-\kappa_{1} x}+\bar{B}_{3} e^{\kappa_{2} x}+\bar{B}_{4} e^{-\kappa_{2} x}+\bar{B}_{5} e^{\kappa_{3} x}+\bar{B}_{6} e^{-\kappa_{3} x} \\
& +\bar{B}_{7} e^{\kappa_{4} x}+\bar{B}_{8} e^{-\kappa_{4} x}=\sum_{j=1}^{4}\left(\bar{B}_{2 j-1} e^{\kappa_{j} x}+\bar{B}_{2 j} e^{-\kappa_{j} x}\right)
\end{aligned}
$$




$$
\begin{aligned}
\bar{\theta}_{1}(x, s)= & \bar{C}_{1} e^{\kappa_{1} x}+\bar{C}_{2} e^{-\kappa_{1} x}+\bar{C}_{3} e^{\kappa_{2} x}+\bar{C}_{4} e^{-\kappa_{2} x}+\bar{C}_{5} e^{\kappa_{3} x}+\bar{C}_{6} e^{-\kappa_{3} x} \\
& +\bar{C}_{7} e^{\kappa_{4} x}+\bar{C}_{8} e^{-\kappa_{4} x}=\sum_{j=1}^{4}\left(\bar{C}_{2 j-1} e^{\kappa_{j} x}+\bar{C}_{2 j} e^{-\kappa_{j} x}\right) \\
\bar{\theta}_{2}(x, s)= & \bar{D}_{1} e^{\kappa_{1} x}+\bar{D}_{2} e^{-\kappa_{1} x}+\bar{D}_{3} e^{\kappa_{2} x}+\bar{D}_{4} e^{-\kappa_{2} x}+\bar{D}_{5} e^{\kappa_{3} x}+\bar{D}_{6} e^{-\kappa_{3} x} \\
& +\bar{D}_{7} e^{\kappa_{4} x}+\bar{D}_{8} e^{-\kappa_{4} x}=\sum_{j=1}^{4}\left(\bar{D}_{2 j-1} e^{\kappa_{j} x}+\bar{D}_{2 j} e^{-\kappa_{j} x}\right),
\end{aligned}
$$

where $\bar{A}_{1}-\bar{A}_{8}, \bar{B}_{1}-\bar{B}_{8}, \bar{C}_{1}-\bar{C}_{8}$, and $\bar{D}_{1}-\bar{D}_{8}$, are four sets of eight constants. $\kappa_{j}=$ $\sqrt{\chi_{j}}(j=1-4), \chi_{j}$ are the roots of

$$
\chi^{4}+a_{1} \chi^{3}+a_{2} \chi^{2}+a_{3} \chi+a_{4}=0
$$

where

$$
a_{1}=\eta_{3} / \eta_{4} \quad a_{2}=\eta_{2} / \eta_{4} \quad a_{3}=\eta_{1} / \eta_{4} \quad a_{4}=\eta_{0} / \eta_{4} .
$$

The fourth-order Equation (9) can be factorized as

$$
\left(\chi^{2}+p_{1} \chi+q_{1}\right)\left(\chi^{2}+p_{2} \chi+q_{2}\right)=0,
$$

where

$$
\left\{\begin{array}{l}
p_{1} \\
p_{2}
\end{array}\right\}=\frac{1}{2}\left[a_{1} \pm \sqrt{a_{1}^{2}-4 a_{2}+4 \lambda_{1}}\right] \quad\left\{\begin{array}{l}
q_{1} \\
q_{2}
\end{array}\right\}=\frac{1}{2}\left[\lambda_{1} \pm \frac{a_{1} \lambda_{1}-2 a_{3}}{\sqrt{a_{1}^{2}-4 a_{2}+4 \lambda_{1}}}\right]
$$

and $\lambda_{1}$ is a real root of the following cubic equation

$$
\lambda^{3}-a_{2} \lambda^{2}+\left(a_{1} a_{3}-4 a_{4}\right) \lambda+\left(4 a_{2} a_{4}-a_{3}^{2}-a_{1}^{2} a_{4}\right)=0 .
$$

Then the four roots of Equation (9) can be written as

$$
\left\{\begin{array}{l}
\chi_{1} \\
\chi_{2}
\end{array}\right\}=-\frac{p_{1}}{2} \pm \sqrt{\frac{p_{1}^{2}}{4}-q_{1}} \quad\left\{\begin{array}{l}
\chi_{3} \\
\chi_{4}
\end{array}\right\}=-\frac{p_{2}}{2} \pm \sqrt{\frac{p_{2}^{2}}{4}-q_{2}} .
$$

In the solution of Equation (9), if any of the $\chi_{j}$ 's are zero or are repeated, the solutions (8) to the differential Equation (7) will be modified according to the well-known methods for ordinary differential equations with constant coefficients, for those particular values of $\chi_{j}$.

From Equation (6a)-(6d), only eight of the thirty-two constants are independent. The relationship among the constants is given by

$$
\begin{array}{cc}
\bar{B}_{2 j-1}=t_{j} \bar{A}_{2 j-1} & \bar{B}_{2 j}=t_{j} \bar{A}_{2 j} \\
\bar{C}_{2 j-1}=\bar{t}_{j} \bar{A}_{2 j-1} \quad \bar{C}_{2 j}=-\bar{t}_{j} \bar{A}_{2 j} \\
\bar{D}_{2 j-1}=\tilde{t}_{j} \bar{A}_{2 j-1} \quad \bar{D}_{2 j}=-\widetilde{t}_{j} \bar{A}_{2 j}
\end{array}
$$


where

$$
\begin{aligned}
t_{j}= & \frac{1}{k}\left[k+P_{1} \kappa_{j}^{2}-A_{1}\left(k_{1} G_{1} \kappa_{j}^{2}-\rho_{1} s^{2}\right)+\frac{k_{1}^{2} G_{1}^{2} A_{1}^{2} \kappa_{j}^{2}}{k_{1} G_{1} A_{1}-I_{1}\left(E_{1} \kappa_{j}^{2}-\rho_{1} s^{2}\right)}\right] \\
\bar{t}_{j}= & \frac{k_{1} G_{1} A_{1} \kappa_{j}}{k_{1} G_{1} A_{1}-I_{1}\left(E_{1} \kappa_{j}^{2}-\rho_{1} s^{2}\right)} \\
\tilde{t}_{j}= & \frac{1}{k_{2} G_{2} A_{2} \kappa_{j}}\left[k+\left(( k + ( - k _ { 2 } G _ { 2 } A _ { 2 } + P _ { 2 } ) \kappa _ { j } ^ { 2 } + \rho _ { 2 } A _ { 2 } s ^ { 2 } ) \left(-k_{1} G_{1} A_{1}^{2} \rho_{1} s^{2}\right.\right.\right. \\
& +I_{1}\left(k+P_{1} \kappa_{j}^{2}\right)\left(E_{1} \kappa_{j}^{2}-\rho_{1} s^{2}\right)-A_{1}\left(k_{1} G_{1}\left(k+P_{1} \kappa_{j}^{2}+E_{1} I_{1} \kappa_{j}^{4}\right)\right. \\
& \left.\left.\left.\left.-\left(E_{1}+k_{1} G_{1}\right) \kappa_{j}^{2} \rho_{1} I_{1} s^{2}+\rho_{1}^{2} I_{1} s^{4}\right)\right)\right) /\left(k\left(k_{1} G_{1} A_{1}-I_{1}\left(E_{1} \kappa_{j}^{2}-\rho_{1} s^{2}\right)\right)\right)\right]
\end{aligned}
$$$$
(j=1-4)
$$

The expressions of shear forces $Q_{1}(x, t), Q_{2}(x, t)$, and bending moments $M_{1}(x, t), M_{2}(x, t)$ can be obtained from Equations (4a)-(4d) as follows:

$$
\begin{gathered}
Q_{1}(x, t)=k_{1} G_{1} A_{1}\left(w_{1}^{\prime}(x, t)-\theta_{1}(x, t)\right)-P_{1} w_{1}^{\prime}(x, t), \\
Q_{2}(x, t)=k_{2} G_{2} A_{2}\left(w_{2}^{\prime}(x, t)-\theta_{2}(x, t)\right)-P_{2} w_{2}^{\prime}(x, t), \\
M_{1}(x, t)=-E_{1} I_{1} \theta_{1}^{\prime}(x, t), \\
M_{2}(x, t)=-E_{2} I_{2} \theta_{2}^{\prime}(x, t) .
\end{gathered}
$$

Application of the Laplace transform on Equation (13a)-(13d) with respect to time $t$ leads to the transformed shear forces $\bar{Q}_{1}(x, s), \bar{Q}_{2}(x, s)$, and the transformed bending moments $\bar{M}_{1}(x, s)$, $\bar{M}_{2}(x, s)$ of the form,

$$
\begin{gathered}
\bar{Q}_{1}(x, s)=k_{1} G_{1} A_{1}\left(\bar{w}_{1}^{\prime}(x, s)-\bar{\theta}_{1}(x, s)\right)-P_{1} \bar{w}_{1}^{\prime}(x, s), \\
\bar{Q}_{2}(x, s)=k_{2} G_{2} A_{2}\left(\bar{w}_{2}^{\prime}(x, s)-\bar{\theta}_{2}(x, s)\right)-P_{2} \bar{w}_{2}^{\prime}(x, s), \\
\bar{M}_{1}(x, s)=-E_{1} I_{1} \bar{\theta}_{1}^{\prime}(x, s), \\
\bar{M}_{2}(x, s)=-E_{2} I_{2} \bar{\theta}_{2}^{\prime}(x, s) .
\end{gathered}
$$

Substitute Equation (8a)-(8d) into Equations (14a)-(14d), the transformed forces and moments at the nodes of the double-beam element (refer to figure 2) are

$$
\begin{gathered}
\bar{Q}_{1}(x=0, s)=\sum_{j=1}^{4}\left[k_{1} G_{1} A_{1}\left(\kappa_{j}-\bar{t}_{j}\right)-P_{1} \kappa_{j}\right]\left(\bar{A}_{2 j-1}-\bar{A}_{2 j}\right) \\
\bar{Q}_{2}(x=0, s)=\sum_{j=1}^{4}\left[k_{2} G_{2} A_{2}\left(t_{j} \kappa_{j}-\tilde{t}_{j}\right)-P_{2} t_{j} \kappa_{j}\right]\left(\bar{A}_{2 j-1}-\bar{A}_{2 j}\right)
\end{gathered}
$$




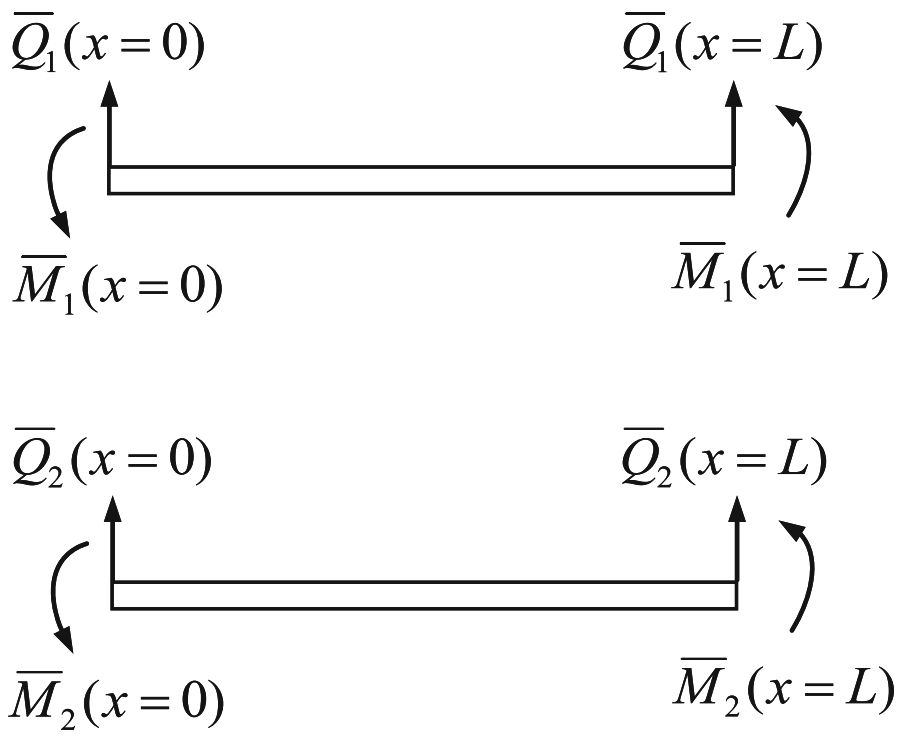

Figure 2. The forces and moments at the nodes of the double-beam element.

$$
\begin{gathered}
\bar{M}_{1}(x=0, s)=\sum_{j=1}^{4}-E_{1} I_{1} \bar{t}_{j} \kappa_{j}\left(\bar{A}_{2 j-1}+\bar{A}_{2 j}\right) \\
\bar{M}_{2}(x=0, s)=\sum_{j=1}^{4}-E_{2} I_{2} \tilde{t}_{j} \kappa_{j}\left(\bar{A}_{2 j-1}+\bar{A}_{2 j}\right) \\
\bar{Q}_{1}(x=L, s)=\sum_{j=1}^{4}\left[k_{1} G_{1} A_{1}\left(\kappa_{j}-\bar{t}_{j}\right)-P_{1} \kappa_{j}\right]\left(\bar{A}_{2 j-1} e^{\kappa_{j} L}-\bar{A}_{2 j} e^{-\kappa_{j} L}\right) \\
\bar{Q}_{2}(x=L, s)=\sum_{j=1}^{4}\left[k_{2} G_{2} A_{2}\left(t_{j} \kappa_{j}-\tilde{t}_{j}\right)-P_{2} t_{j} \kappa_{j}\right]\left(\bar{A}_{2 j-1} e^{\kappa_{j} L}-\bar{A}_{2 j} e^{-\kappa_{j} L}\right) \\
\bar{M}_{1}(x=L, s)=\sum_{j=1}^{4}-E_{1} I_{1} \bar{t}_{j} \kappa_{j}\left(\bar{A}_{2 j-1} e^{\kappa_{j} L}+\bar{A}_{2 j} e^{-\kappa_{j} L}\right) \\
\bar{M}_{2}(x=L, s)=\sum_{j=1}^{4}-E_{2} I_{2} \tilde{t}_{j} \kappa_{j}\left(\bar{A}_{2 j-1} e^{\kappa_{j} L}+\bar{A}_{2 j} e^{-\kappa_{j} L}\right) .
\end{gathered}
$$

The four generalized displacement components, being $\bar{w}_{1}(x, s), \bar{w}_{2}(x, s), \bar{\theta}_{1}(x, s)$, and $\bar{\theta}_{2}(x, s)$, contribute to four sets of stiffness influence coefficients at each node, a total of eight sets for this double-beam element. Any one of the set of stiffness influence coefficients can be obtained by computing the forces and moments in the transformed domain required at nodes to maintain a unit value of transformed displacement at a particular degree of freedom (DOF) while the other seven are fixed. Thus, the eight sets of influence coefficients can be obtained by solving for parameters $A_{2 j-1}$ and $A_{2 j}(j=1-4)$ for the eight states, given below. The substitution of $A_{2 j-1}$ and $A_{2 j}(j=1-4)$ from each of these states in Equations (15a)-(15h) generates the associated 
forces or moments in the transform domain which are the required dynamic stiffness influence coefficients for that DOF.

The eight sets of motion configuration states for the eight DOFs at double-beam nodes are

(1) $\bar{w}_{1}(0, s)=1, \bar{w}_{2}(0, s)=\bar{\theta}_{1}(0, s)=\bar{\theta}_{2}(0, s)=0$

$$
\bar{w}_{1}(L, s)=\bar{w}_{2}(L, s)=\bar{\theta}_{1}(L, s)=\bar{\theta}_{2}(L, s)=0
$$

(2) $\bar{w}_{2}(0, s)=1, \bar{w}_{1}(0, s)=\bar{\theta}_{1}(0, s)=\bar{\theta}_{2}(0, s)=0$

$$
\bar{w}_{1}(L, s)=\bar{w}_{2}(L, s)=\bar{\theta}_{1}(L, s)=\bar{\theta}_{2}(L, s)=0
$$

(3) $\bar{\theta}_{1}(0, s)=1, \bar{w}_{1}(0, s)=\bar{w}_{2}(0, s)=\bar{\theta}_{2}(0, s)=0$

$$
\bar{w}_{1}(L, s)=\bar{w}_{2}(L, s)=\bar{\theta}_{1}(L, s)=\bar{\theta}_{2}(L, s)=0
$$

(4) $\bar{\theta}_{2}(0, s)=1, \bar{w}_{1}(0, s)=\bar{w}_{2}(0, s)=\bar{\theta}_{1}(0, s)=0$

$$
\bar{w}_{1}(L, s)=\bar{w}_{2}(L, s)=\bar{\theta}_{1}(L, s)=\bar{\theta}_{2}(L, s)=0
$$

(5) $\bar{w}_{1}(L, s)=1, \bar{w}_{2}(L, s)=\bar{\theta}_{1}(L, s)=\bar{\theta}_{2}(L, s)=0$

$$
\bar{w}_{1}(0, s)=\bar{w}_{2}(0, s)=\bar{\theta}_{1}(0, s)=\bar{\theta}_{2}(0, s)=0
$$

(6) $\bar{w}_{2}(L, s)=1, \bar{w}_{1}(L, s)=\bar{\theta}_{1}(L, s)=\bar{\theta}_{2}(L, s)=0$

$$
\bar{w}_{1}(0, s)=\bar{w}_{2}(0, s)=\bar{\theta}_{1}(0, s)=\bar{\theta}_{2}(0, s)=0
$$

(7) $\bar{\theta}_{1}(L, s)=1, \bar{w}_{1}(L, s)=\bar{w}_{2}(L, s)=\bar{\theta}_{2}(L, s)=0$

$$
\bar{w}_{1}(0, s)=\bar{w}_{2}(0, s)=\bar{\theta}_{1}(0, s)=\bar{\theta}_{2}(0, s)=0
$$

(8) $\bar{\theta}_{2}(L, s)=1, \bar{w}_{1}(L, s)=\bar{w}_{2}(L, s)=\bar{\theta}_{1}(L, s)=0$

$$
\bar{w}_{1}(0, s)=\bar{w}_{2}(0, s)=\bar{\theta}_{1}(0, s)=\bar{\theta}_{2}(0, s)=0 .
$$

The determination of the stiffness influence coefficients seems very tedious; however, it can be done conveniently by using the symbolic manipulator software such as Maple (Char et al 1988). Such a process of obtaining the dynamic stiffness influence coefficients can result in the exact analytical stiffness terms for the uniform double-beam element. The explicit expressions of the dynamic stiffness influence coefficients are too lengthy to list in the paper.

Such computed dynamic stiffness matrix $[\bar{K}(s)]$ is the transformed dynamic stiffness matrix in the Laplace domain. The Laplace transform parameter $s$ is related to the frequency $\omega$ by $s=i \omega$, i.e., the frequency dynamic stiffness matrix $[\bar{K}(\omega)]$ is obtained by using $s=i \omega$. The frequency dynamic stiffness matrix can now be used to compute the natural frequencies of either an individual double-beam system or an assembly of double-beam systems. A reliable and efficient Wittrick-Williams algorithm (Wittrick \& Williams 1971) is adopted in the present study to obtain the natural frequencies of the double-beam system. The algorithm does not directly compute the natural frequencies, but instead simply finds the total number of natural frequencies below an arbitrarily given trial value. In this way, the upper and lower bounds are established 
Table 1. Natural frequency $(\mathrm{Hz})$ of the axially loaded double-beam system for Case I.

\begin{tabular}{|c|c|c|c|c|c|c|}
\hline \multirow[t]{2}{*}{ Mode no. } & \multicolumn{2}{|c|}{$P_{1}=0$ and $P_{2}=0$} & \multicolumn{2}{|c|}{$P_{1}=800 \mathrm{~N}$ and $P_{2}=1000 \mathrm{~N}$} & \multicolumn{2}{|c|}{$P_{1}=1000 \mathrm{~N}$ and $P_{2}=1500 \mathrm{~N}$} \\
\hline & $\overline{\text { Present }}$ & ANSYS & Present & ANSYS & Present & ANSYS \\
\hline 1 & 38.14 & 38.14 & 28.52 & 28.52 & 25.16 & 25.16 \\
\hline 2 & 60.13 & 60.14 & 56.31 & 56.31 & 54.47 & 54.48 \\
\hline 3 & 79.41 & 79.42 & 61.89 & 61.90 & 56.59 & 56.60 \\
\hline 4 & 146.04 & 146.04 & 126.69 & 126.69 & 121.37 & 121.37 \\
\hline 5 & 147.08 & 147.08 & 141.76 & 141.76 & 139.02 & 139.02 \\
\hline 6 & 237.51 & 237.52 & 217.41 & 217.41 & 212.08 & 212.09 \\
\hline
\end{tabular}

on each required natural frequency, after which the well-known bisection method can be used to determine the natural frequencies to any required accuracy. Since the extensive discussions can be found in the literature related to the use of the algorithm, the procedure is not repeated here. The mode shapes corresponding to the natural frequencies can be found in the usual way by making an arbitrary assumption about one unknown variable of the double-beam system and then calculating the remaining variables in terms of the arbitrarily chosen one.

\section{Example and results}

In this example, the properties of an axially loaded double-beam system to be used in the numerical calculations are:

$$
\begin{aligned}
& b_{1}=0.01 \mathrm{~m}, h_{1}=0.005 \mathrm{~m}, b_{2}=0.01 \mathrm{~m}, h_{2}=0.01 \mathrm{~m}, L=1 \mathrm{~m}, E_{1}=2.0 \times 10^{11} \mathrm{~N} / \mathrm{m}^{2}, \\
& E_{2}=2.0 \times 10^{11} \mathrm{~N} / \mathrm{m}^{2}, v_{1}=0.3, v_{2}=0.3, k_{1}=0.87, k_{2}=0.87, \rho_{1}=7600.0 \mathrm{~kg} / \mathrm{m}^{3}, \\
& \rho_{2}=7600.0 \mathrm{~kg} / \mathrm{m}^{3}, k=16.0 \times 10^{3} \mathrm{~N} / \mathrm{m}^{2} .
\end{aligned}
$$

Here, $b_{i}$ and $h_{i}$ denote the width and height of the beam, respectively. $v_{i}$ denotes the Poisson ratio of the beam material. The area of the beam cross-section $A_{i}=b_{i} h_{i}$. The second moment of area of the beam section $I_{i}=1 / 12 b_{i} h_{i}^{3}$.

A detailed free vibration analysis of the axially loaded double-beam system is now performed for ten interesting cases of the different boundary conditions.

Case I: upper beam clamped-clamped, lower beam clamped-clamped;

\begin{tabular}{|c|c|c|c|c|c|c|}
\hline \multirow[t]{2}{*}{ Mode no. } & \multicolumn{2}{|c|}{$P_{1}=0$ and $P_{2}=0$} & \multicolumn{2}{|c|}{$P_{1}=800 \mathrm{~N}$ and $P_{2}=1000 \mathrm{~N}$} & \multicolumn{2}{|c|}{$P_{1}=1000 \mathrm{~N}$ and $P_{2}=1500 \mathrm{~N}$} \\
\hline & Present & ANSYS & Present & ANSYS & $\overline{\text { Present }}$ & ANSYS \\
\hline 1 & 28.92 & 28.93 & 16.57 & 16.57 & 9.45 & 9.46 \\
\hline 2 & 49.11 & 49.12 & 43.69 & 43.69 & 39.20 & 39.20 \\
\hline 3 & 66.88 & 66.89 & 47.18 & 47.18 & 42.91 & 42.92 \\
\hline 4 & 120.11 & 120.12 & 105.43 & 105.44 & 99.32 & 99.33 \\
\hline 5 & 127.00 & 127.00 & 113.99 & 114.00 & 110.82 & 110.82 \\
\hline 6 & 212.41 & 212.42 & 190.60 & 190.61 & 184.75 & 184.75 \\
\hline
\end{tabular}

Case II: upper beam clamped-simply supported, lower beam clamped-simply supported;

Table 2. Natural frequency $(\mathrm{Hz})$ of the axially loaded double-beam system for Case II. 
Table 3. Natural frequency (Hz) of the axially loaded double-beam system for Case III.

\begin{tabular}{lrrrrrrrr}
\hline Mode no. & \multicolumn{2}{c}{$P_{1}=0$ and $P_{2}=0$} & & \multicolumn{2}{c}{$P_{1}=100 \mathrm{~N}$ and $P_{2}=150 \mathrm{~N}$} & & \multicolumn{2}{c}{$P_{1}=150 \mathrm{~N}$ and $P_{2}=200 \mathrm{~N}$} \\
\cline { 2 - 3 } & Present & ANSYS & & Present & ANSYS & & Present & ANSYS \\
\hline 1 & 7.15 & 7.15 & & 4.86 & 4.87 & & 3.37 & 3.38 \\
2 & 37.74 & 37.85 & & 34.79 & 34.97 & & 33.19 & 33.41 \\
3 & 40.42 & 40.83 & & 40.09 & 40.48 & & 39.94 & 40.33 \\
4 & 59.52 & 59.72 & & 58.06 & 58.27 & & 57.51 & 57.72 \\
5 & 79.44 & 79.58 & & 76.14 & 76.27 & & 74.44 & 74.57 \\
6 & 146.05 & 146.13 & & 142.75 & 142.83 & & 141.08 & 141.16 \\
\hline
\end{tabular}

Table 4. Natural frequency (Hz) of the axially loaded double-beam system for Case IV.

\begin{tabular}{|c|c|c|c|c|c|c|}
\hline \multirow[t]{2}{*}{ Mode no. } & \multicolumn{2}{|c|}{$P_{1}=0$ and $P_{2}=0$} & \multicolumn{2}{|c|}{$P_{1}=400 \mathrm{~N}$ and $P_{2}=600 \mathrm{~N}$} & \multicolumn{2}{|c|}{$P_{1}=600 \mathrm{~N}$ and $P_{2}=800 \mathrm{~N}$} \\
\hline & Present & ANSYS & Present & ANSYS & Present & ANSYS \\
\hline 1 & 19.52 & 19.52 & 12.36 & 12.37 & 7.41 & 7.41 \\
\hline 2 & 43.52 & 43.53 & 40.77 & 40.77 & 39.53 & 39.54 \\
\hline 3 & 56.00 & 56.00 & 45.68 & 45.69 & 39.55 & 39.56 \\
\hline 4 & 96.29 & 96.30 & 92.08 & 92.09 & 90.62 & 90.63 \\
\hline 5 & 109.53 & 109.53 & 98.13 & 98.13 & 91.90 & 91.91 \\
\hline 6 & 188.80 & 188.80 & 177.30 & 177.30 & 171.26 & 171.27 \\
\hline
\end{tabular}

Table 5. Natural frequency $(\mathrm{Hz})$ of the axially loaded double-beam system for Case V.

\begin{tabular}{|c|c|c|c|c|c|c|}
\hline \multirow[t]{2}{*}{ Mode no. } & \multicolumn{2}{|c|}{$P_{1}=0$ and $P_{2}=0$} & \multicolumn{2}{|c|}{$P_{1}=350 \mathrm{~N}$ and $P_{2}=500 \mathrm{~N}$} & \multicolumn{2}{|c|}{$P_{1}=400 \mathrm{~N}$ and $P_{2}=800 \mathrm{~N}$} \\
\hline & $\overline{\text { Present }}$ & ANSYS & Present & ANSYS & $\overline{\text { Present }}$ & ANSYS \\
\hline 1 & 18.76 & 18.80 & 12.04 & 12.22 & 6.11 & 6.80 \\
\hline 2 & 31.98 & 32.47 & 25.77 & 26.80 & 23.22 & 24.27 \\
\hline 3 & 39.22 & 39.73 & 26.61 & 27.36 & 25.49 & 26.32 \\
\hline 4 & 44.76 & 44.92 & 41.40 & 41.41 & 40.79 & 40.82 \\
\hline 5 & 79.18 & 79.46 & 61.05 & 61.38 & 57.97 & 58.30 \\
\hline 6 & 96.41 & 96.42 & 92.82 & 92.83 & 90.69 & 90.69 \\
\hline
\end{tabular}

Table 6. Natural frequency (Hz) of the axially loaded double-beam system for Case VI.

\begin{tabular}{|c|c|c|c|c|c|c|}
\hline \multirow[t]{2}{*}{ Mode no. } & \multicolumn{2}{|c|}{$P_{1}=0$ and $P_{2}=0$} & \multicolumn{2}{|c|}{$P_{1}=350 \mathrm{~N}$ and $P_{2}=500 \mathrm{~N}$} & \multicolumn{2}{|c|}{$P_{1}=400 \mathrm{~N}$ and $P_{2}=800 \mathrm{~N}$} \\
\hline & Present & ANSYS & Present & ANSYS & Present & ANSYS \\
\hline 1 & 25.69 & 25.76 & 19.10 & 19.56 & 14.68 & 15.54 \\
\hline 2 & 32.32 & 32.79 & 26.43 & 27.24 & 25.09 & 25.91 \\
\hline 3 & 40.37 & 40.97 & 28.25 & 28.93 & 27.30 & 27.95 \\
\hline 4 & 48.79 & 48.83 & 46.19 & 46.21 & 45.23 & 45.24 \\
\hline 5 & 79.47 & 79.76 & 61.33 & 61.66 & 58.30 & 58.62 \\
\hline 6 & 120.09 & 120.10 & 117.05 & 117.05 & 115.20 & 115.21 \\
\hline
\end{tabular}


Table 7. Natural frequency (Hz) of the axially loaded double-beam system for Case VII.

\begin{tabular}{lrrrrrrrr}
\hline Mode no. & \multicolumn{2}{c}{$P_{1}=0$ and $P_{2}=0$} & & \multicolumn{2}{c}{$P_{1}=350 \mathrm{~N}$ and $P_{2}=500 \mathrm{~N}$} & & \multicolumn{2}{c}{$P_{1}=400 \mathrm{~N}$ and $P_{2}=800 \mathrm{~N}$} \\
\cline { 2 - 3 } & Present & ANSYS & & Present & ANSYS & & Present & ANSYS \\
\hline 1 & 29.82 & 29.90 & & 21.61 & 22.40 & & 17.08 & 18.20 \\
2 & 32.46 & 32.95 & & 27.13 & 27.88 & & 26.03 & 26.86 \\
3 & 41.03 & 41.62 & & 30.28 & 30.70 & & 30.02 & 30.38 \\
4 & 59.68 & 59.69 & & 57.77 & 57.77 & & 56.75 & 56.76 \\
5 & 79.52 & 79.81 & & 61.35 & 61.68 & & 58.31 & 58.64 \\
6 & 146.08 & 146.23 & 130.28 & 130.45 & & 127.86 & 128.03 \\
\hline
\end{tabular}

Table 8. Natural frequency $(\mathrm{Hz})$ of the axially loaded double-beam system for Case VIII.

\begin{tabular}{lrrrrrrrr}
\hline Mode no. & \multicolumn{2}{c}{$P_{1}=0$ and $P_{2}=0$} & & \multicolumn{2}{c}{$P_{1}=400 \mathrm{~N}$ and $P_{2}=600 \mathrm{~N}$} & & \multicolumn{2}{c}{$P_{1}=600 \mathrm{~N}$ and $P_{2}=800 \mathrm{~N}$} \\
\cline { 2 - 3 } & Present & ANSYS & & Present & ANSYS & & Present & ANSYS \\
\hline 1 & 16.08 & 16.28 & & 10.92 & 11.21 & 6.91 & 7.35 \\
2 & 36.46 & 36.54 & & 31.71 & 31.83 & & 28.97 & 29.11 \\
3 & 55.89 & 55.91 & & 47.20 & 47.20 & & 41.99 & 42.01 \\
4 & 59.25 & 59.32 & & 52.86 & 52.94 & & 50.76 & 50.84 \\
5 & 109.64 & 109.65 & & 98.27 & 98.28 & & 92.07 & 92.07 \\
6 & 147.10 & 147.14 & 141.75 & 141.80 & & 139.93 & 139.97 \\
\hline
\end{tabular}

Table 9. Natural frequency $(\mathrm{Hz})$ of the axially loaded double-beam system for Case IX.

\begin{tabular}{|c|c|c|c|c|c|c|}
\hline \multirow[t]{2}{*}{ Mode no. } & \multicolumn{2}{|c|}{$P_{1}=0$ and $P_{2}=0$} & \multicolumn{2}{|c|}{$P_{1}=800 \mathrm{~N}$ and $P_{2}=1000 \mathrm{~N}$} & \multicolumn{2}{|c|}{$P_{1}=1000 \mathrm{~N}$ and $P_{2}=1500 \mathrm{~N}$} \\
\hline & Present & ANSYS & Present & ANSYS & Present & ANSYS \\
\hline 1 & 26.80 & 26.81 & 15.11 & 15.12 & 8.57 & 8.57 \\
\hline 2 & 48.07 & 48.07 & 32.61 & 32.61 & 23.46 & 23.46 \\
\hline 3 & 56.63 & 56.64 & 43.93 & 43.93 & 41.77 & 41.77 \\
\hline 4 & 109.50 & 109.50 & 85.22 & 85.23 & 77.97 & 77.97 \\
\hline 5 & 120.13 & 120.13 & 113.98 & 113.99 & 110.80 & 110.81 \\
\hline 6 & 188.80 & 188.80 & 165.00 & 165.01 & 158.50 & 158.50 \\
\hline
\end{tabular}

Table 10. Natural frequency $(\mathrm{Hz})$ of the axially loaded double-beam system for Case X.

\begin{tabular}{|c|c|c|c|c|c|c|}
\hline \multirow[t]{2}{*}{ Mode no. } & \multicolumn{2}{|c|}{$P_{1}=0$ and $P_{2}=0$} & \multicolumn{2}{|c|}{$P_{1}=800 \mathrm{~N}$ and $P_{2}=1000 \mathrm{~N}$} & \multicolumn{2}{|c|}{$P_{1}=1000 \mathrm{~N}$ and $P_{2}=1500 \mathrm{~N}$} \\
\hline & $\overline{\text { Present }}$ & ANSYS & Present & ANSYS & Present & ANSYS \\
\hline 1 & 31.07 & 31.07 & 21.20 & 21.20 & 17.65 & 17.65 \\
\hline 2 & 56.57 & 56.57 & 33.11 & 33.11 & 23.86 & 23.87 \\
\hline 3 & 59.53 & 59.54 & 55.86 & 55.86 & 54.02 & 54.03 \\
\hline 4 & 109.58 & 109.59 & 85.29 & 85.30 & 78.05 & 78.06 \\
\hline 5 & 147.05 & 147.05 & 141.72 & 141.72 & 138.98 & 138.98 \\
\hline 6 & 188.81 & 188.81 & 165.02 & 165.03 & 158.52 & 158.52 \\
\hline
\end{tabular}



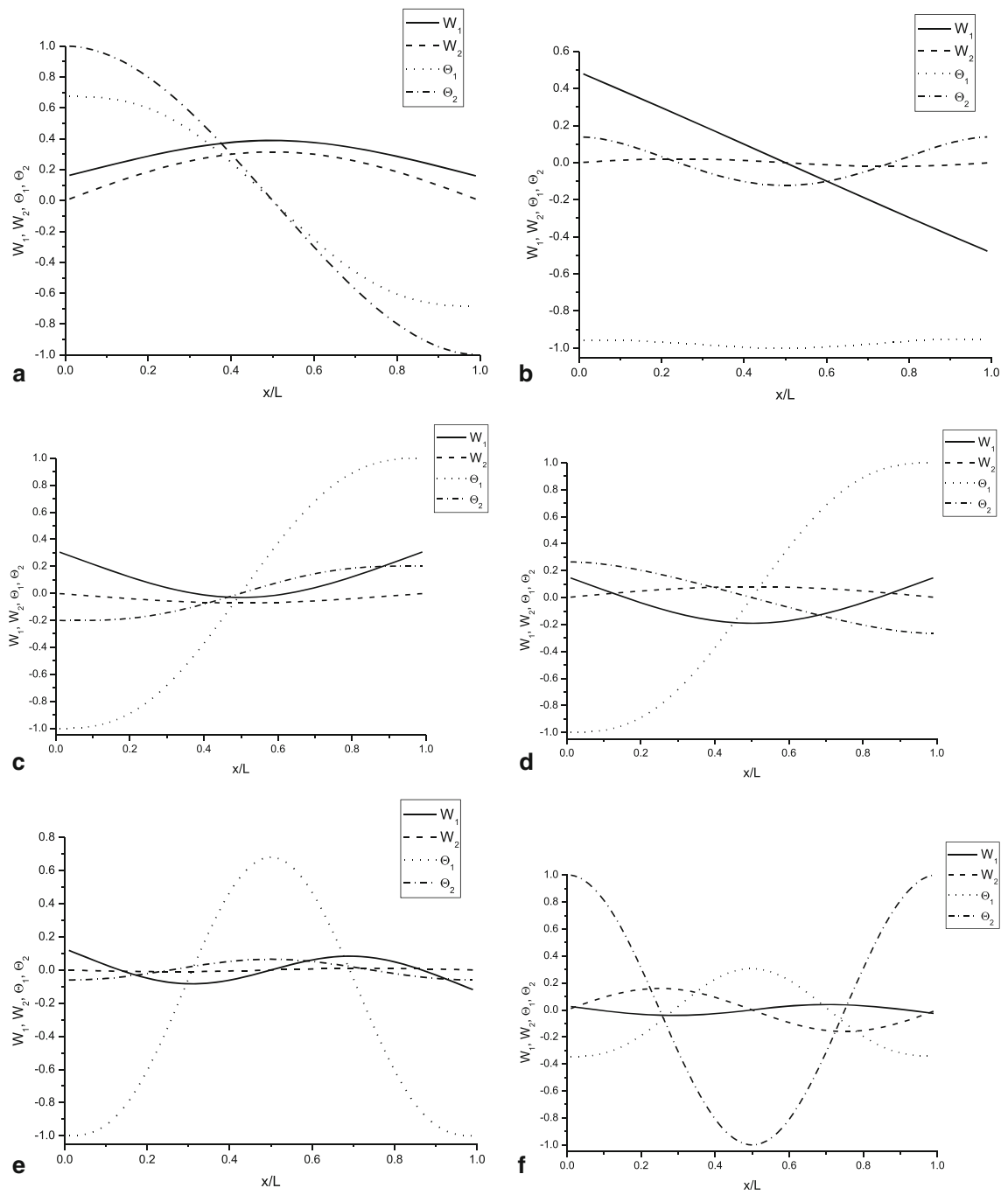

Figure 3. The first six normal mode shapes of the double-beam system for Case $\mathrm{V}$ with $P_{1}=0$ and $P_{2}=$ 0: (a) mode 1; (b) mode 2; (c) mode 3; (d) mode 4; (e) mode 5; (f) mode 6.

Case III: upper beam clamped-free, lower beam clamped-free beam;

Case IV: upper beam simply supported-simply supported, lower beam simply supportedsimply supported;

Case V: upper beam free-free, lower beam simply supported-simply supported;

Case VI: upper beam free-free, lower beam clamped-simply supported;

Case VII: upper beam free-free, lower beam clamped-clamped;

Case VIII: upper beam simply supported-simply supported, lower beam clamped-free;

Case IX: upper beam simply supported-simply supported, lower beam clamped-simply supported;

Case X: upper beam simply supported-simply supported, lower beam clamped-clamped. 

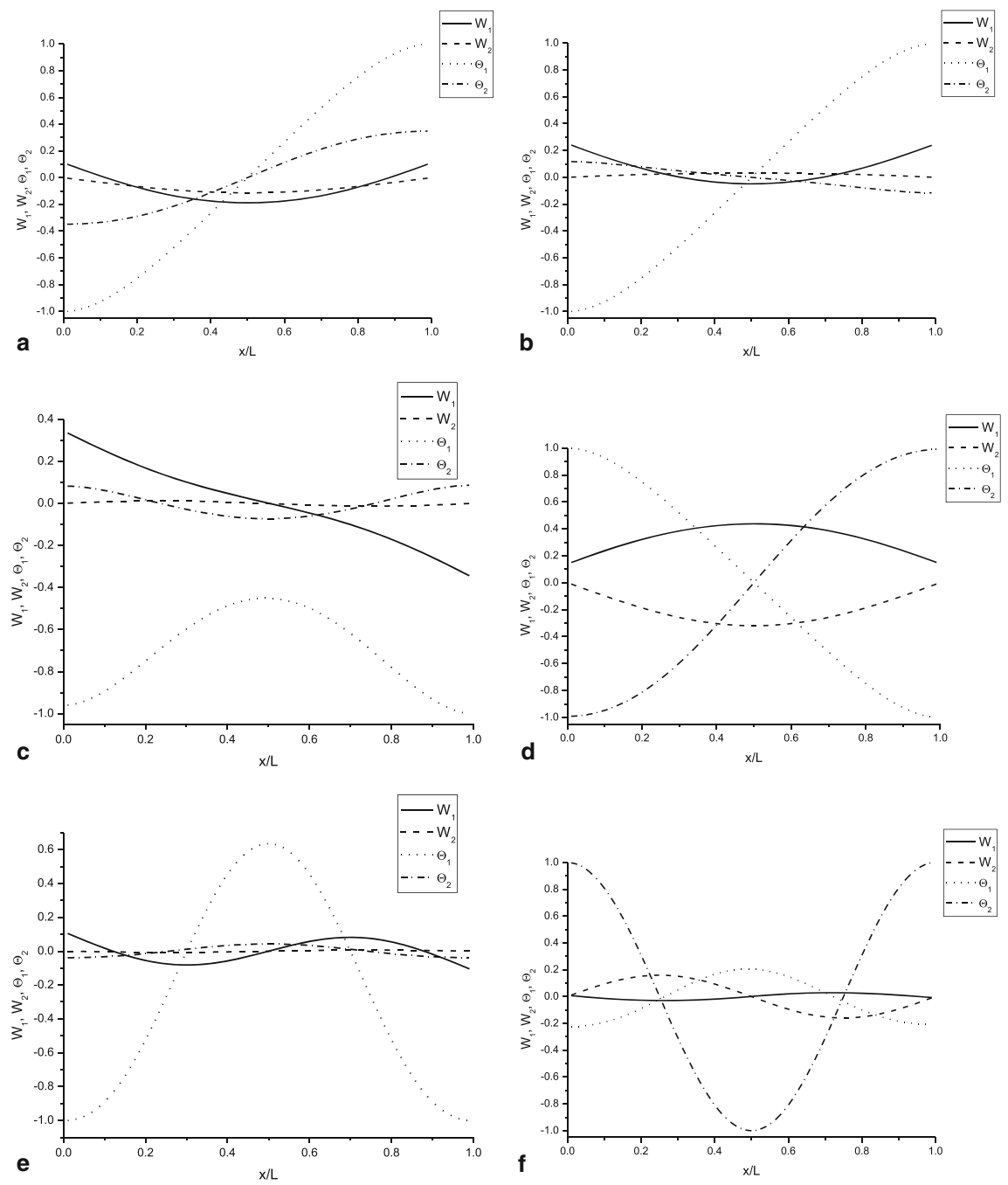

Figure 4. The first six normal mode shapes of the double-beam system for Case $\mathrm{V}$ with $P_{1}=350 \mathrm{~N}$ and $P_{2}=500 \mathrm{~N}$ : (a) mode 1; (b) mode 2; (c) mode 3; (d) mode 4; (e) mode 5; (f) mode 6.

The first six natural frequencies of the double-beam system are calculated by using the exact dynamic stiffness formulation proposed in this paper, and the numerical results are given in tables 1-10. In each case three different axial forces are considered. Tables 1-10 can be used to investigate the effect of the axial forces $P_{1}$ and $P_{2}$ on the natural frequencies of the doublebeam system. Only the compressive axial forces are considered in this study. It should be noted that letting $P_{1}=0$ and $P_{2}=0$ yields the solutions for the unloaded double-beam system. In order to validate the accuracy and efficiency of the proposed dynamic stiffness formulation, the corresponding natural frequencies calculated by use of the commercial software ANSYS are also displayed in tables 1-10 as reference. The element selected in calculating the natural frequencies in ANSYS is a two-node beam element BEAM3 with 3-DOF at each node. The first-order shear 

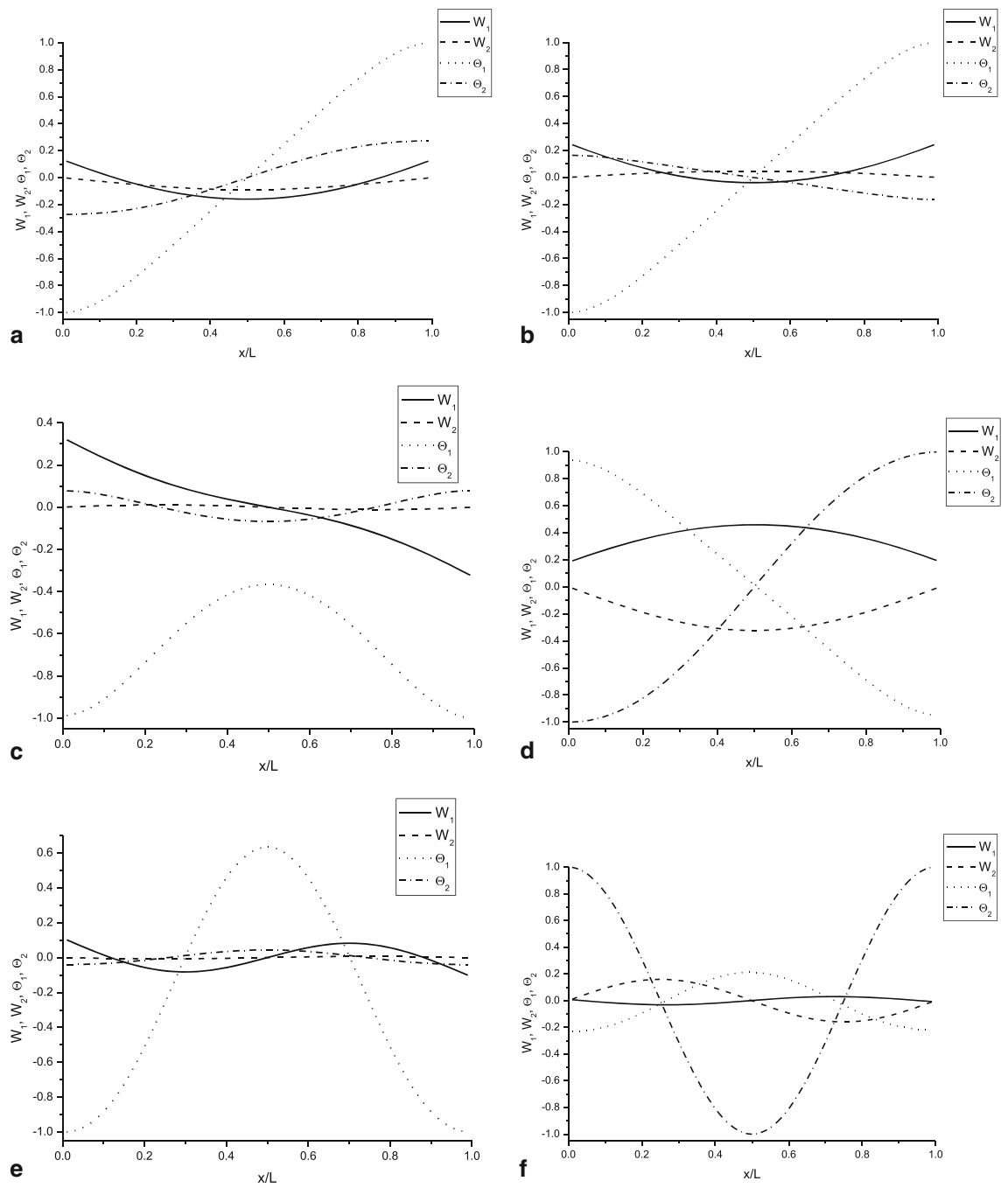

Figure 5. The first six normal mode shapes of the double-beam system for Case $\mathrm{V}$ with $P_{1}=400 \mathrm{~N}$ and $P_{2}=800 \mathrm{~N}$ : (a) mode $1 ;(\mathbf{b})$ mode $2 ;(\mathbf{c})$ mode $3 ;(\mathbf{d})$ mode $4 ;(\mathbf{e})$ mode $5 ;$ (f) mode 6.

deformation beam theory is adopted in the formulation of the beam element. The element mesh used in the ANSYS model is $2 \times 100$ in the whole double-beam system.

It can be seen from tables 1-10 that the present results agree closely with the solutions obtained from ANSYS. It is also evident from the numerical results shown in tables 1-10 that the natural frequencies of the double-beam system are sensitive to the variations of the axial force. As expected, the natural frequencies excluding the effect of axial force are higher than the ones including the effect of axial force. All the natural frequencies become lower as the axial force becomes larger. The effect of the axial force on the fundamental natural frequency is significant for all ten cases. From the numerical analysis it is seen that there is a general tendency 
to decrease the natural frequencies in the case of increasing the axial force. The decrease in the lower natural frequencies is generally greater than that in the higher ones.

Further, the effect of the axial forces $P_{1}$ and $P_{2}$ on the normal mode shapes of the doublebeam system is studied. The normal mode shapes for Case $\mathrm{V}$ with three different axial forces considered are computed and illustrated in figures 3-5. The first six normal mode shapes for the unloaded case are plotted in figures 3(a)-(f). The first six normal mode shapes for the case of axial forces $P_{1}=350 \mathrm{~N}$ and $P_{2}=500 \mathrm{~N}$ are shown in figures 4(a)-(f). The first six normal mode shapes for the case of axial forces $P_{1}=400 \mathrm{~N}$ and $P_{2}=800 \mathrm{~N}$ are illustrated in figures 5(a)-(f).

It is clear from figures 3-5 that the axial force has a prominent effect on the mode shapes of the double-beam system, especially for the lower modes.

\section{Conclusions}

The dynamic stiffness method to analyse the free vibration of the axially loaded double-beam systems considering the effects of shear deformation and rotary inertia has been successfully proposed in this paper. Firstly, the Laplace transform method is used to formulate the dynamic stiffness influence coefficients by directly solving the governing differential equations in the transform domain. Then, an example is given to study the effect of the axial force on the natural frequencies and mode shapes. It shows that substantial differences may be incurred if the axial force is ignored, especially for the lower modes. This method can provide an efficient and accurate means for the dynamic analysis of the axially loaded double-beam systems. In addition, this dynamic stiffness method may also be applied to the forced vibration analysis of the axially loaded double-beam systems.

\section{References}

Abu-Hilal M 2006 Dynamic response of a double Euler-Bernoulli beam due to a moving constant load. $J$. Sound Vib. 297: 477-491

Aida T, Toda S, Ogawa N and Imada Y 1992 Vibration control of beams by beam-type dynamic vibration absorbers. J. Eng. Mech. 118: 248-258

Balkaya M, Kaya M O and Saglamer A 2010 Free transverse vibrations of an elastically connected simply supported twin pipe system. Struct. Eng. Mech. 34: 549-561

Char B W, Geddes K O, Gonnet G H, Monagan M B and Watt S M 1988 Maple reference manual (Canada: Watcom Publications)

Chen Y H and Sheu J T 1994 Dynamic characteristics of layered beam with flexible core. J. Vib. Acoust. 116: $350-356$

Chen Y H and Lin C Y 1998 Structural analysis and optimal design of a dynamic absorbing beam. J. Sound Vib. 212: 759-769

Chonan S 1976 Dynamical behaviours of elastically connected double-beam systems subjected to an impulsive load. T. JSME 19: 595-603

Hamada T R, Nakayama H and Hayashi K 1983 Free and forced vibrations of elastically connected doublebeam systems. T. JSME 26: 1936-1942

Kessel P G 1966 Resonances excited in an elastically connected double-beam system by a cyclic moving load. J. Acoust. Soc. Am. 40: 684-687

Oniszczuk Z 2000 Free transverse vibrations of elastically connected simply supported double-beam complex system. J. Sound Vib. 232: 387-403

Oniszczuk Z 2003 Forced transverse vibrations of an elastically connected complex simply supported double-beam system. J. Sound Vib. 264: 273-286 
Palmeri A and Adhikari S 2011 A Galerkin-type state-space approach for transverse vibrations of slender double-beam systems with viscoelastic inner layer. J. Sound Vib. 330: 6372-6386

Rao S S 1974 Natural vibrations of systems of elastically connected Timoshenko beams. J. Acoust. Soc. Am. 55: 1232-1237

Seelig J M and Hoppmann II W H 1964 Normal mode vibrations of systems of elastically connected parallel bars. J. Acoust. Soc. Am. 36: 93-99

Vu H V, Ordonez A M and Karnopp B H 2000 Vibration of a double-beam system. J. Sound Vib. 229: 807822

Wittrick W H and Williams F W 1971 A general algorithm for computing natural frequencies of elastic structures. Q. J. Mech. Appl. Math 24: 263-284

Zhang Y Q, Lu Y, Wang S L and Liu X 2008 Vibration and buckling of a double-beam system under compressive axial loading. J. Sound Vib. 318: 341-352 